\title{
Shear-induced diffusion: the role of granular clusters
}

\author{
Matthew Macaulay ${ }^{1, *}$ and Pierre Rognon ${ }^{1, * *}$ \\ ${ }^{1}$ Particles and Grains laboratory, School of Civil Engineering, The University of Sydney. Building J05, 2006 Sydney Australia.
}

\begin{abstract}
This paper is concerned with the physical mechanisms controlling shear-induced diffusion in dense granular flows. The starting point is that of the granular random walk occurring in diluted granular flows, which underpins Bagnold's scaling relating the coefficient of self-diffusion to the grain size and shear rate. By means of DEM simulations of plane shear flows, we measure some deviations from this scaling in dense granular flows with and without contact adhesion. We propose to relate these deviations to the development of correlated motion of grains in these flows, which impacts the magnitude of grain velocity fluctuations and their time persistence.
\end{abstract}

\section{Introduction}

In granular flows, grains do not strictly follow the main streamlines. Rather, their trajectories exhibit some degree of randomness. This results in a process known as shearinduced diffusion [1-5]. Figure 1 illustrates this process in the case of a plane shear flow: when a packing is subjected to some shear deformation, grains diffuse in the direction transverse to shear. Shear-induced diffusion is a fundamental feature of granular flows. It is a direct driver of grain mixing, which enhances the transfer of heat [6] and counterbalances potential segregation effects [7].

Granular materials involve grains that are large enough to be athermal. Unlike colloids or molecules, they do not diffuse under the effect of thermal fluctuations. Rather, grains can only diffuse if the material is being deformed under the action of some external mechanical loading. In shear flows the grain size $d$ and the shear rate $\dot{\gamma}$ are two elementary scales from which one can dimensionally build a macroscopic coefficient of diffusion $D$ [1-5]:

$$
D \propto d^{2} \dot{\gamma}
$$

We refer to this law as Bagnold's scaling. According to Einstein's theory for a random walk, this scaling is consistent with a simple characteristic trajectory: grains making steps of size $d$ in a random direction at a frequency $\dot{\gamma}$. This scaling is verified in diluted granular flows, where the grains' trajectory indeed involves a series of free, ballistic flights interrupted by a binary collision with another grain.

However, deviations from that scaling were observed in dense granular flows of cohesionless and cohesive grains [8-10]. A qualitative way to understand the origin of these deviations is that grains interact via multiple and sustained contacts in dense granular flows. This means that they are not likely to experience a ballistic

\footnotetext{
*e-mail: matthew.macaulay@sydney.edu.au

**e-mail: pierre.rognon@sydney.edu.au

A video is available at https://doi.org/10.48448/9sd2-hg49
}

flight, which implies no contact, during an entire unit shear deformation. As a result of these interactions, the kinematic field of dense granular flows features large velocity fluctuations which are correlated in space and in time [1117]. This reflects the spontaneous development of transient clusters of grains, which move as a quasi-rigid body during a finite period of time

These clusters introduce a meso-length scale $\ell$ that is larger than a grain and a characteristic time scale that is that of their lifetime $T$. From a purely dimensional point of view, one could expect that these scales could be involved in the Bagnold's scaling, perhaps in conjunction with $d$ and $\dot{\gamma}$.

The purpose of this paper is to analyse the deviation from the Bagnold's scaling for diffusivity in dense granular materials using the perspective of the clusters. In this aim, we synthesise previously published results focusing on (i) cohesionless flows at various inertial numbers [8] and (ii) cohesive granular flows [10]. The underlying reasoning is that tuning these two control parameters (inertial number and cohesion) affects the size and the life-time of the clusters in different ways and that these variations will highlight how $\ell$ and $T$ might affect the diffusivity $D$.

\section{Method}

To measure both the macroscopic diffusivity and the internal kinematic in granular flow, we used a numerical approach based on a discrete element method. The flow configuration is that presented on figure 1: a pseudobidimensional packing of $100 \times 100$ grains is subjected to a plane shear flow, where both the shear rate $\dot{\gamma}$ and the normal stress $P$ are prescribed. Pseudo-bidimensional means here that grains are all placed in the same plan $\{x, y\}$ and never move in the $z$-direction. However, they are considered to be cylinders with a diameter $d$ and depth $d$ in the $z$-direction. 


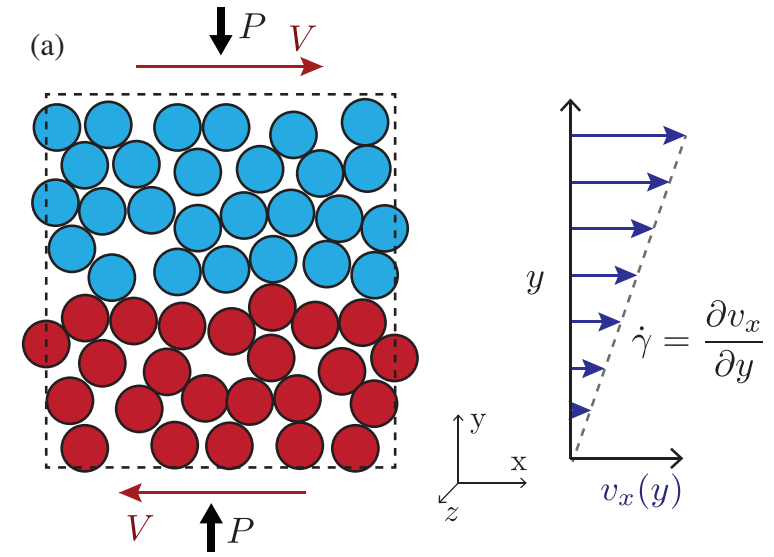

(b)

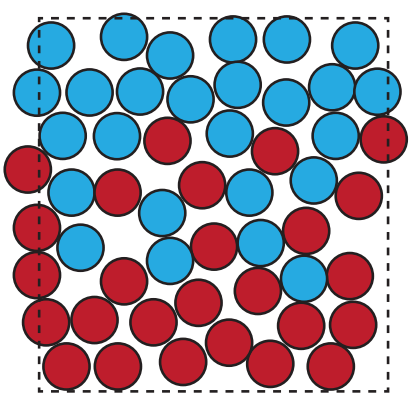

Figure 1. Illustration of mixing resulting from shear-induced diffusion. An assembly of grains is sheared in the $x$-direction by applying opposite velocities $V$ at the top and bottom and a normal stress $P$. (a) Initially, grains located in the bottom half of the shear cell are coloured in red and those in the top-half in blue; grains keep their colour during the flow. (b) After some shear deformation, a mixing zone develops in the centre of the cell. The DEM simulations presented in the paper simulate this configuration: plane shear flow prescribing the normal stress $P$ and the shear rate $\dot{\gamma}$, using periodic boundary conditions (represented with the dashed line). This configuration leads to a homogenous shear state characterised by the linear velocity profile represented here, where $v_{x}(y)$ is the average velocity of the grains located at the height $y$.

A polydispersity of $d \pm 20 \%$ on the grain diameter is introduced to prevent crystallisation during shear. Grains have a density $\rho$. They interact by direct contact, with no long-range interactions. Contact forces involve a tangential Coulomb friction, a normal linear (Hookean) elastic repulsion of stiffness $k$, a normal viscous dissipation and a normal constant adhesion force $f_{0}$. The contact parameters are set to the following values: the contact friction coefficient is 0.5 , the normal stiffness $k /(P d)$ is $10^{4}$, the viscous damping coefficient is such that a binary collision has a coefficient of restitution of 0.5 in absence of adhesion. The effect of these contact parameter on the rheological property of granular flow is thoroughly discussed in Refs. [18-22].

The two dimensionless numbers that will be varied in the following are the inertial number $I$ and the cohesion number $C$, defined as:

$$
I=\dot{\gamma} t_{i} ; \quad t_{i}=d \sqrt{\frac{\rho}{P}} ; \quad C=\frac{f_{0}}{P d^{2}} .
$$

The inertial number will be varied from $5.10^{-3}$ to 0.5 , a range corresponding to the dense flow regime [18, 23]. $C$, which measures the intensity of the contact tensile strength, will be varied from 0 to $10[16,17] . \quad C=0$ corresponds to a cohesionless material. The maximum of $C=10$ is chosen as we observed that higher values sometimes lead to heterogeneous shear flows featuring a shear band, which are not compatible with the analysis presented below.

Selecting a value for $I$ and $C$ in these ranges, we obtained steady and homogenous shear flows characterised by statistically invariant stress and shear rate both in space and in time. In particular, the use of periodic boundary conditions in both directions prevents flow heterogeneities that a solid wall would typically produce $[24,25]$. Taking advantage of the ergodicity of such flows, we measured various quantities of interest considering both the entire shear cell and the entire duration of the flow (50 shear deformation). These quantities include the grain vertical mean square displacement $\Delta$, the grain velocity fluctuation $\delta v$ and the time persistence of these velocity fluctuations $\Psi$, defined as:

$$
\begin{aligned}
\Delta(\tau) & =\left\langle\left(y_{i}(t+\tau)-y_{i}(t)\right)^{2}\right\rangle \\
\delta v & =\sqrt{\left\langle v_{i}^{2}\right\rangle-\left\langle v_{i}\right\rangle^{2}} ; \\
\Psi & =\int_{t^{\prime}=0}^{\infty} \frac{\left\langle\delta v(t) \delta v\left(t+t^{\prime}\right)\right\rangle}{\delta v^{2}} d t^{\prime} .
\end{aligned}
$$

$y_{i}$ and $v_{i}$ are the vertical component of the position and of the velocity of grain $i$ at a given time, and the angle brackets represent the average operator considering all grains and times $t$. The time $\Psi$ is the integral of the autocorrelation function of the grain velocity. It measures how long, on average, the grain velocity remains nearly constant. More details on how these quantities are measured can be found in Ref. [8, 10].

\section{Deviation from the Bagnold's scaling}

Figure 2 presents measurements of the diffusivity $D$ obtained by using the Einstein relation:

$$
D=\frac{1}{2} \lim _{\tau \rightarrow \infty} \frac{\Delta(\tau)}{\tau} .
$$

The first observation is that cohesion enhances the diffusivity, regardless of the inertial number. Within the explored range of parameters, this enhancement is significant: it can reach an order of magnitude. The second observation is that the Bagnold's scaling is recovered at high 


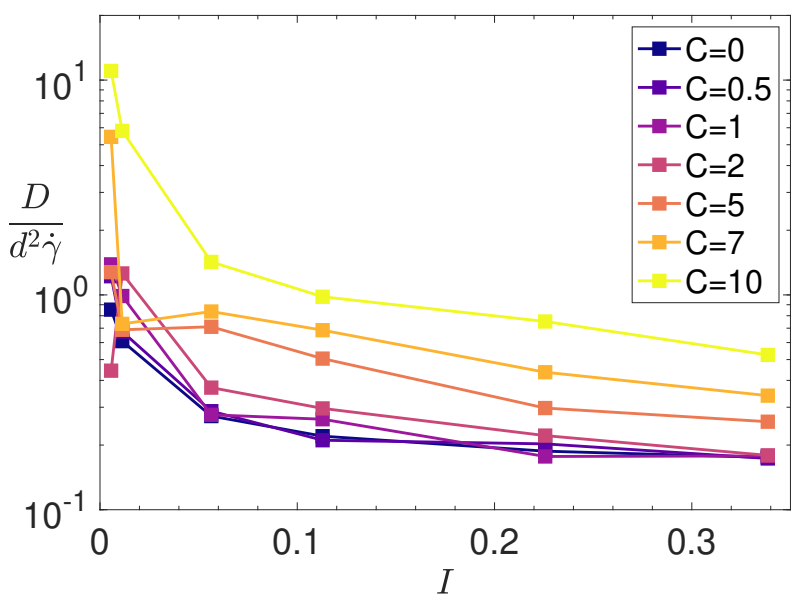

Figure 2. Diffusivity $D$ measured using (6) in flows with different inertial numbers $I$ and cohesion number $C$.

inertial number $\left(\frac{D}{\dot{\gamma} d^{2}}\right.$ is then nearly independent of $\left.I\right)$, both for cohesionless and cohesive grains. In contrast, there is a deviation from that scaling at low inertial numbers: the diffusivity becomes dependent of $I$ and the ratio $\frac{D}{\dot{\gamma} d^{2}}$ increases as the inertial number decreases.

\section{Insights from Green-Kubo relation}

In order to understand the origin of these deviations, we consider the Green-Kubo relation that expresses the diffusivity in terms of the velocity fluctuation and their time persistence:

$$
D=\delta v^{2} \Psi
$$

This relation points out that having a large diffusivity requires the velocity fluctuation to have a large magnitude and to be sustained in time.

Figure 3 shows the evolution of $\delta v$ and $\Psi$ with the inertial number for various level of cohesion. Results suggest the following scalings:

$$
\frac{\delta v}{\dot{\gamma} d} \approx \alpha(C) \frac{1}{I^{0.5}} \text { and } \frac{\Psi}{t_{i}} \approx \beta(C) \frac{1}{I^{0.75}} .
$$

The effect of the cohesion is to increase both the magnitude of the velocity fluctuations and their persistence time, by increasing the coefficient $\alpha$ and $\beta$. Result suggests a linear increase $\alpha(C) \approx 0.3+0.04 C$ and $\beta(C) \approx$ $0.22+0.019 C$.

Figure 4 confirms the validity of the scaling 7 by comparing the diffusivities measured using (6) to $\delta v^{2} \Psi$.

\section{A cluster based diffusivity scaling}

To connect these results to the existence of clusters in granular flows, we consider the results presented in Ref. [26]. This study considered the mixing of passive tracers in a velocity field comprised of a rotating vortex of size $\ell_{v}$, rotating for a period of time $t_{v}$ at an angular velocity $w$ before randomly changing position. This produces
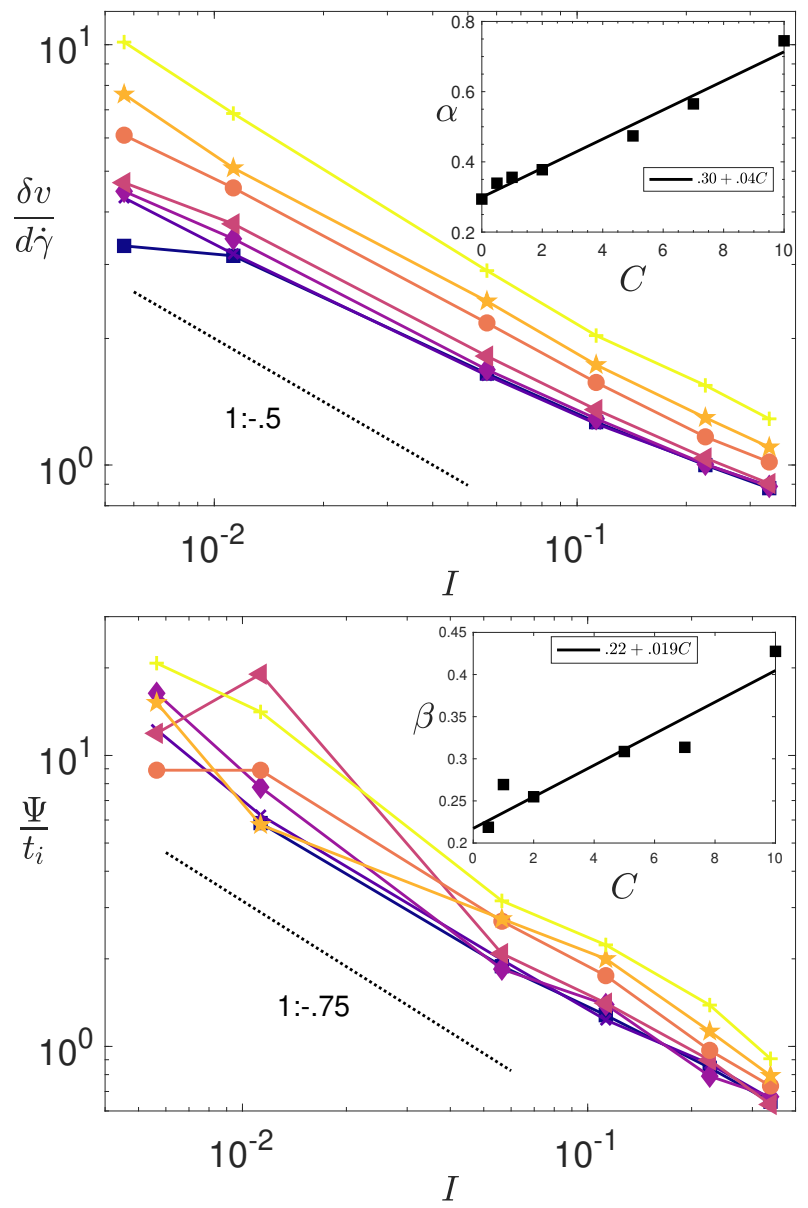

Figure 3. Velocity fluctuation $\delta v$ (a) and their time persistence $\Psi$ (b) measured in flows with different inertial numbers $I$ and cohesion number $C$.The dashed lines represent a power low with an exponent -0.5 and -0.75 for visual reference. Symbols represent different levels of cohesion as per figure 2. Insets show the coefficient $\alpha$ and $\beta$ obtained by fitting the data with Eq. (8), as well as linear fits for $\alpha(C)$ and $\beta(C)$ (solid lines).

some chaotic advection and induces the mixing of tracers. The result of this study is that the effective diffusivity of the tracers subjected to this advective field scales like $\left(\ell_{v} w t_{v}\right)^{2} / t_{v}$. This is consistent with the following trajectory of tracers at the edge of a vortex: they move over a step of size $\ell_{v} w t_{v}$ in a time step $t_{v}$ before the vortex changes position.

Drawing an analogy between the mixing of grains and the mixing these tracers, we identify (i) $t_{v}$ to the velocity correlation time $\Psi$, implying that $\Psi$ measures a typical granular cluster lifetime $T, w$ to $\dot{\gamma}$ which measures the scale of vorticity in the flow and (iii) $\ell_{v}$ to $\delta v / \dot{\gamma}$. This implies a relation between the velocity fluctuation and a typical granular cluster size $\ell=\delta v / \dot{\gamma}$. This relation is compatible with a cluster rotating as a rigid body at a rotation rate $\dot{\gamma}$, which yields a velocity for grains at the edge of the cluster proportional to $\ell \dot{\gamma}$.

Accordingly, we propose to express the shear-induced diffusivity in terms of cluster size and lifetime as:

$$
D=(\ell \dot{\gamma} T)^{2} / T
$$




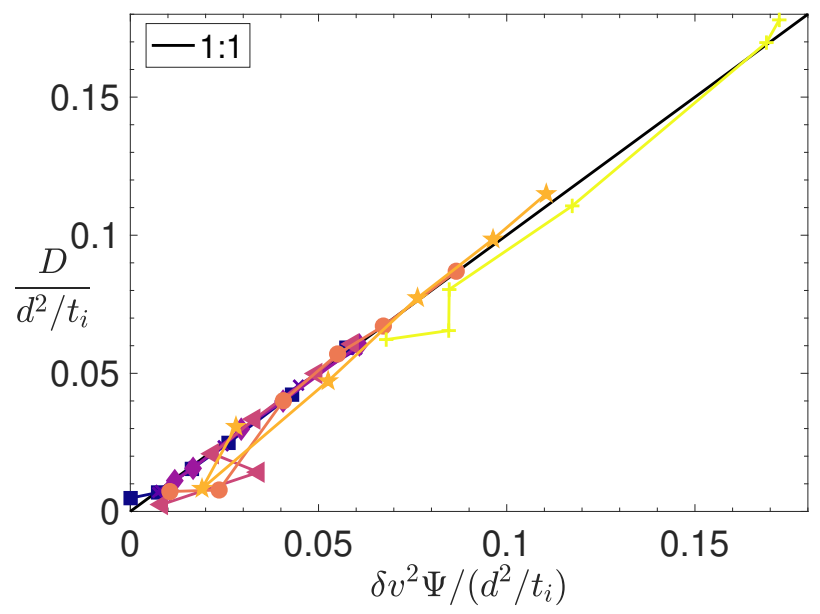

Figure 4. Diffusivity $D$ measured using (6) compared with the product $\delta v^{2} \Psi$. The dashed lines represent the unity function for visual reference. Symbols represent different level of cohesion as per figure 2 .

Relating $\ell$ and $T$ to the velocity fluctuations and their time persistence $(\ell=\delta v / \dot{\gamma}$ and $T=\Psi)$ yields $D=\delta v^{2} \Psi$, which corresponds to the Green-Kubo relation (7). Using the scaling in Eq (8) enables us to translate this expression to an expression for the diffusivity in terms of the two dimensionless control numbers $I$ and $C$ :

$$
D \approx \alpha^{2} \frac{d^{2} \dot{\gamma}^{2}}{I} \frac{\beta t_{i}}{I^{\frac{3}{4}}}=d^{2} \dot{\gamma} \frac{\alpha^{2} \beta}{I^{\frac{3}{4}}}
$$

This expression highlights how the inertial number $I$ and the cohesion $C$ (via the parameters $\alpha(C)$ and $\beta(C)$ ) affect Bagnold's scaling of shear-induced diffusivity in dense granular flows.

\section{Conclusion}

The results and analysis presented here provide information at two levels.

Firstly, at the macroscopic level, they point out that the diffusivity in dense granular flow generally deviates from Bagnold's scaling (1). This results from a contribution from the inertial number and the cohesion number in the presence of adhesion. The scaling (10) that we proposed to capture the diffusivity may readily be used in continuum models for granular flows provided that the two controlling dimensionless numbers $I$ and $C$ are known.

Secondly, they explain the microscopic origin of this macroscopic diffusivity, in terms of velocity fluctuation and their time persistence, or equivalently in terms of cluster size and lifetime. This understanding may be useful to further analyse the shear-induced behaviour of similar materials including dense suspensions, foams and emulsions, which may exhibit a similar internal kinematic.

\section{References}

[1] C.S. Campbell, J. Fluid Mech. 348, 85 (1997)

[2] S.S. Hsiau, Y.M. Shieh, Chem. Eng. Sci. 55, 1969 (2000)

[3] S.S. Hsiau, S.C. Yang, Chem. Eng. Sci 58, 339 (2003)

[4] B. Utter, R.P. Behringer, Phys. Rev. E 69, 031308 (2004)

[5] S.S. Hsiau, L.S. Lu, C.Y. Chou, W.L. Yang, Int. J. Multiph. Flow 34, 352 (2008)

[6] P. Rognon, I. Einav, Phys. Rev. Lett. 105, 218301 (2010)

[7] J. Ottino, D. Khakhar, Annu. Rev. Fluid Mech. 32, 55 (2000)

[8] P. Kharel, P. Rognon, Phys. Rev. Lett. 119, 178001 (2017)

[9] P. Kharel, P. Rognon, EPL (Europhysics Letters) 124, 24002 (2018)

[10] M. Macaulay, P. Rognon, J. Fluid Mech. 858 (2019)

[11] F. Radjai, S. Roux, Phys. Rev. Lett. 89, 064302 (2002)

[12] O. Pouliquen, Phys. Rev. Lett. 93, 248001 (2004)

[13] H.H. Shen, B. Sankaran, Phys. Rev. E 70, 051308 (2004)

[14] E. DeGiuli, J. McElwaine, M. Wyart, Phys. Rev. E 94, 012904 (2016)

[15] P. Rognon, T. Miller, I. Einav, Granul. Matter 17, 177 (2015)

[16] P.G. Rognon, J.N. Roux, M. Naaim, F. Chevoir, J. Fluid Mech. 596, 21 (2008)

[17] P. Rognon, J.N. Roux, D. Wolf, M. Naaïm, F. Chevoir, EPL 74, 644 (2006)

[18] F. Da Cruz, S. Emam, M. Prochnow, J.N. Roux, F. Chevoir, Phys. Rev. E 72, 021309 (2005)

[19] S. Roy, S. Luding, T. Weinhart, New J. Phys. 19, 043014 (2017)

[20] S. Mandal, M. Nicolas, O. Pouliquen, PNAS 117, 8366 (2020)

[21] H. Shi, S. Roy, T. Weinhart, V. Magnanimo, S. Luding, Granul. Matter 22, 14 (2019)

[22] T.T. Vo, S. Nezamabadi, P. Mutabaruka, J.Y. Delenne, F. Radjai, Nat. Commun. 11, 1 (2020)

[23] G. MiDi, EPJE 14, 341 (2004)

[24] T. Miller, P. Rognon, B. Metzger, I. Einav, Phys. Rev. Lett. 111, 058002 (2013)

[25] P.G. Rognon, T. Miller, B. Metzger, I. Einav, J. Fluid Mech. 764, 171 (2015)

[26] D. Griffani, P. Rognon, B. Metzger, I. Einav, Phys. Fluids 25, 093301 (2013) 